\title{
Principles of managing patients with personality disorder
}

\author{
Sophie E. Davison
}

The management of individuals with personality disorder is one of the most challenging and sometimes controversial areas of psychiatry. This paper describes the principles involved in identifying the clinical problems and formulating a management plan for patients with personality disorder in everyday clinical practice. It demonstrates that the principles of assessing and managing personality disorders and the clinical skills required are no different from those of treating any other chronic mental disorder.

Patients with personality disorder have multiple and diverse needs. Some present repeatedly to services. Although there are a number of difficulties in managing patients with personality disorder, their problems are easier to tackle if the patients are properly assessed, their individual needs identified and an appropriate plan formulated.

\section{Personality disorder is a mental disorder}

One reason that personality disorders may not be diagnosed is the misconception that they are not mental disorders (see Adshead, 2001 for a discussion of the concepts of disease, illness and disorder as they relate to personality disorder).

The ICD-10 definition of a mental disorder refers to:

"the existence of a recognisable set of symptoms and behaviours in most cases associated with distress and interference with social function" (World Health Organization, 1992: p. 5).
The definition of a personality disorder in DSMIV (American Psychiatric Association, 1994: p. 633). includes the following characteristics:

"an enduring pattern of inner experience and behavior that deviates markedly from the expectations of the individual's culture. This pattern is manifested in two or more of the following areas:

(1) cognition (i.e., ways of perceiving and interpreting self, other people and events)

(2) affectivity (i.e., the range, intensity, lability, and appropriateness of emotional response)

(3) interpersonal functioning

(4) impulse control".

The pattern must be inflexible and pervasive across a broad range of personal and social situations. For a diagnosis to be made, the enduring pattern of inner experience and behaviour (i.e. the symptoms) must lead to clinically significant distress or impairment in social, occupational or other important areas of functioning.

Thus, the term mental disorder applies as much to personality disorder as it does to Axis I disorders such as schizophrenia. Individuals may need help as a result of their distress or because their symptoms are interfering with their everyday functioning. Personality disorders are associated with high levels of dysfunction, comparable to major Axis I disorders (Nakao et al, 1992).

There are many disorders (e.g. anxiety and depression) for which the border between normality and abnormality is not always clear-cut. This does not lead clinicians to argue that the diagnosis should not be made. The cut-off is a pragmatic one. Individuals generally present to services when their symptoms cause distress or start to interfere with

Sophie Davison is a clinical lecturer in forensic psychiatry at the Institute of Psychiatry (Department of Forensic Psychiatry, Institute of Psychiatry, De Crespigny Park, London SE5 8AF, UK; e-mail: s.davison@iop.kcl.ac.uk) and consultant forensic psychiatrist at Broadmoor Hospital, Crowthorne, Berkshire. Her research interests are in the clinical presentation and management of personality disorder in different settings. 
functioning. This is when it is generally deemed appropriate for services to intervene.

\section{Personality in clinical practice}

Some clinicians regard the assessment and management of personality and personality disorders as a specialist subject about which they know little or nothing. However, personality affects all doctorpatient interactions even if it is not addressed explicitly. It affects help-seeking behaviours, compliance with treatment, coping styles, social networks, therapeutic alliance, risk-taking behaviour and lifestyle (Box 1). These factors in turn affect the risk of developing a number of physical and psychiatric disorders, as well as their prognosis and management.

For example, the management plan drawn up for a patient with schizophrenia who is compliant with treatment, has formed a good therapeutic relationship with his or her community mental health team and has a large network of supportive friends and family to alert professionals of relapse may differ from that for a patient who is non-compliant, uses illicit drugs, only turns up in crisis, has lost contact with all social support and whose hostel placements keep breaking down because of antisocial behaviour. Another example is a residential placement that breaks down for one elderly person but not for another with exactly the same degree of cognitive impairment: the breakdown may relate to personality factors.

\section{Personality disorder in clinical practice}

Mental health services all have considerable experience of dealing with patients with personality disorders. Many patients presenting to psychiatric services suffer from a personality disorder, although relatively few are explicitly being treated for it. Patients with personality disorders may well present for the first time for treatment of comorbid Axis I disorders.

Having a personality disorder during adolescence doubles the risk of having anxiety, mood disorders, self-harming behaviour and substance use disorders during early adulthood (Johnson et al, 1999). Many patients being treated for Axis I disorders suffer from comorbid personality disorders. Exact rates depend on the population studied (for an overview see de Girolamo \& Reich, 1993). Patients with affective

\section{Box 1 Effects of personality on physical and} mental illness

Personality affects the prognosis, management and risk of developing many physical and mental disorders by its effect on:

Help-seeking behaviours

Compliance with treatment

Coping styles

Risk-taking

Lifestyle

Social support networks

Therapeutic alliance

Eating and drinking habits

Smoking habits

Sexual habits

disorders have high rates of borderline, antisocial, avoidant and dependent personality disorder (de Girolamo \& Reich, 1993). Personality disorders have also been reported in association with eating disorders, somatisation disorders, schizophrenia, bipolar disorders, sexual disorders, obsessivecompulsive disorders and dissociative disorders (Dowson \& Grounds, 1995). It has been suggested that 'difficult' patients with a mixture of antisocial, borderline, narcissistic and histrionic traits are particularly likely to present with depressive syndromes, episodes of self-harm, anxiety states, substance misuse and eating disorders (Higgitt \& Fonagy, 1992).

In primary care settings, Casey \& Tyrer (1990) found that about a third of people attending general practitioners (GPs) had a personality disorder. For the vast majority it was not assessed by the GPs as the primary reason for presenting. A number present as problematic medical patients (Emerson et al, 1994).

Studies using research diagnostic instruments have found that $20-40 \%$ of psychiatric out-patients and about $50 \%$ of psychiatric in-patients fulfil criteria for a personality disorder (for overviews see: de Girolamo \& Reich, 1993; Dowson \& Grounds, 1995; Moran, 1999). However, this is rarely the primary focus of treatment. National Health Service (NHS) hospital in-patient data for the period from 1 April 1998 to 31 March 1999 showed that the primary diagnosis was personality disorder in only 9954 (4\%) of 243039 completed in-patient admissions for treatment of mental disorders (Department of Health, 2000a). In 1999/2000, only 95 (0.3\%) out of 25302 compulsory admissions under the Mental Health Act 1983 were under the category of psychopathic disorder (Department of Health, 2000b). 
Individuals with personality disorders, particularly of the antisocial, paranoid and borderline types, frequently present within a criminal justice context. They account for a large proportion of assessments of patients detained by police under Section 136 of the Mental Health Act 1983 in innercity areas (Spence \& McPhillips, 1995). A survey of psychiatric morbidity in prisons in England and Wales estimated that $78 \%$ of male remand prisoners, $64 \%$ of male sentenced prisoners and $50 \%$ of female prisoners had a personality disorder (Singleton et al, 1998). Within high secure forensic psychiatric services there is much overlap in the clinical needs of those with a primary diagnosis of personality disorder and those admitted for treatment of a mental illness such as schizophrenia (Maden et al, 1995). All have similar needs for social skills training, anger management and drug and alcohol treatment.

\section{The burden of personality disorder}

Personality disorders have a significant impact on mental health services and patient management, even when they are not the primary focus of treatment (Box 2). Patients with comorbid personality disorders have more severe Axis I symptomatology (Tyrer et al, 1990). Patients with major depression, panic disorder and obsessive-compulsive disorder who have a comorbid personality disorder show a poorer response to a range of treatments (Reich \& Green, 1991). Patients with comorbid psychotic disorders and personality disorders are among the heaviest users of psychiatric services (Kent et al, 1995). A community survey found that individuals in the community with evidence of personality disorder make more out-patient mental health visits and have more hospital admissions than those without personality disorder. The more severe the personality disorder pathology the greater the utilisation of mental health services (Reich et al,

Box 2 Common characteristics of patients with personality disorder

High rates of Axis I psychiatric disorders

More severe Axis I symptoms

Worse prognosis of Axis I disorders

Longer and costlier treatments for Axis I disorders

Excess mortality from suicide, accidents and violence
1989). Menzies et al (1993) found that individuals with personality disorders were frequent users of mental health and criminal justice services in the year prior to treatment in a therapeutic community.

Personality disorders have public health importance by virtue of their association with mental illness, eating and drinking habits, smoking, accidents and sexual behaviour. Patients with personality disorder, especially of the antisocial and borderline cluster, have higher rates of suicide and accidental deaths than the general population (Dowson \& Grounds, 1995). Some of the traits associated with the antisocial cluster (cluster B) personality disorders such as impulsivity and recklessness may contribute to high rates of physical morbidity owing to drug use, alcohol use, violence, self-harm and risk-taking behaviours.

\section{Treatability}

It would appear that the concept of 'treatability' in the context of personality disorder arose from the criteria for compulsory admission of patients with the disorder. These state that treatment in hospital must be likely to alleviate the condition or prevent deterioration. Treatability is, in fact, a very broad concept but it has been used to exclude patients not only from compulsory admission but also from other services. Some argue that personality disorders are untreatable and therefore services have little to offer. The debate gets polarised into treatability versus untreatability. This is oversimplistic and often masks a moral debate about responsibility for one's predicament and about who is deserving of treatment (Dowson \& Grounds, 1995). Gwen Adshead's (2001) contribution to this edition explores the concept of treatability in more detail.

As Adshead clearly describes, in no other branch of medicine does treatable equate with curable. Furthermore, within psychiatry doctors do not give up on patients with other chronic enduring mental illnesses on the grounds that they cannot be cured. For example, much time and effort are put into services for 'treatment-resistant' schizophrenia. The principles of treatment are those of treating any chronic condition. The underlying conditions cannot always be eradicated but distress and impairment in functioning may be alleviated in some areas. Associated conditions may be treated. Aggravating and perpetuating factors may be manipulated.

In fact, research suggests that outcome, particularly for patients with borderline personality disorder, is much better than is usually assumed (for review see Sanislow \& McGlashan, 1998), with over half showing clinical recovery at 10-25-year 
follow-up. A recent follow-up of offender patients from high-security hospitals in England and Wales found that those with personality disorder did better in terms of psychosocial outcomes than did those with other mental disorders, usually schizophrenia (Steels et al, 1998).

\section{Developing a framework for assessment in routine clinical practice}

There is increasing emphasis, particularly in relation to research, on using standardised assessments. These can be useful in ensuring consistency and in ensuring that all the different areas of abnormal personality functioning have been asked about. However, many instruments produce a categorical ICD-10 or DSM-IV diagnosis. In clinical terms, simply knowing that an individual fulfils criteria for a particular DSM-IV or ICD-10 personality disorder does not provide the accurate information needed about the individual's actual difficulties and circumstances that is required to plan and prioritise interventions. The principle is the same as in other areas of psychiatry. Giving a patient a label of schizophrenia gives the clinician only a very limited amount of information about his or her individual treatment needs.

Most experienced clinicians make the diagnosis of personality disorder by taking a systematic history and listening to patients' narratives of their lives. Patients tell the story of their relationships with family, loved ones, friends, authorities and colleagues at home, at work, at leisure and at school. Clinicians look for recurring patterns of behaviour and interpersonal interactions, from which they draw inferences about characteristic patterns of relating to others, behaviour patterns, coping mechanisms, belief patterns, hopes, fears and emotional responses.

\section{Functional assessment}

For practical clinical purposes, it has been suggested that the most useful approach is to undertake a functional assessment of personality (Box 3). This is essentially a case formulation that lists the relevant areas of abnormal personality (Gunn, 1993; Westen \& Arkowitz-Westen, 1998) and then examines the distress and functional disability caused by these abnormalities. This process makes the task of drawing up a management plan less daunting. It provides a clearer picture of the therapeutic task, by

separating out different problems, which can each be considered in their own right (Gunn, 1993).

Gunn suggests listing the abnormal traits under the headings: thinking; feelings and emotions; behaviour; social functions; and insight. The 'thinking' heading would include the patients' beliefs about themselves (e.g. low self-esteem, a sense of entitlement out of proportion to the situation) and their beliefs about others (e.g. everyone is hostile and untrustworthy). 'Feelings and emotions' would include any abnormalities in the quality or intensity of emotions, such as outbursts of inappropriate anger, rapidly fluctuating mood, persistent low mood, anxiety, low tolerance of frustration. Social functioning relates to interpersonal problems such as the inability to trust others and the tendency to invite rejection, to become dependent or to form unstable intense relationships.

Once the problem traits have been identified one can assess the associated distress. This may amount to a comorbid psychiatric disorder, such as depression, substance misuse, psychosis or anxiety, or to more non-specific physical or psychological symptoms.

Next, the interference with functioning (or disability, to use World Health Organization (2001) terminology) can be described. A useful framework would be to look at occupational functioning, family functioning (including functioning in relation to partner and to children) and social functioning (housing, finance, social relationships, crime). Each patient's social context is unique and must be assessed and understood.

In completing the functional analysis it is therefore helpful to try to gain an understanding of the circumstances in which individual difficulties occur. In particular, it is useful to clarify what predisposing, precipitating and perpetuating or Box 3 Key tasks of functional analysis
(Gunn, 1993)

List abnormal personality features:

Thoughts

Feelings and emotions

Behaviour

Social (interpersonal) functioning

Insight

Describe associated distress, including comorbid Axis I disorders

Describe interference with functioning:

Occupational

Family

Social 
aggravating factors there are; to understand how the various difficulties interact; and to try to understand the relative importance of different factors in contributing to risk, distress and interference with function. Although complicated, it is the same process as used in assessing other mental disorders.

\section{Formulating a management plan}

Once the problems have been listed and 'unpicked' in this way it becomes possible to make a realistic appraisal of what can and cannot be done. It may be feasible to help improve functioning and reduce distress in some areas but not others. The areas for intervention and goals can be prioritised. This enables professionals to determine whether the right services and resources are available; to assess who has the correct expertise to help with particular aspects of the problem; and to determine the balance between their responsibility towards the patient and towards the public. In this way the limits to treatment are clarified and clear boundaries of responsibility demarcated. If it is not possible to meet a particular need, the reasons can be made explicit and justified.

\section{Key features of a successful management plan}

The contents of the management plan will depend on the assessment of needs. A few key principles have been identified consistently as more likely to be successful in the management of patients with personality disorder (Box 4).

The most important factor is to have explicit and realistic goals in treatment (Box 5). These may be very modest in terms of expected cognitive or personality change. However, this should not discourage the therapist, as even a modest change can have major beneficial effects on social functioning and harm reduction. For example it may not be possible to prevent a patient with antisocial personality disorder from offending but it may be possible to reduce the impact. Long-term personality change is likely to be an appropriate goal only in a few highly motivated patients.

Goals must be prioritised to prevent the treatment task from becoming too daunting. It has to be acknowledged that it may not be possible to intervene in all areas. Goals may change over time. There should also be a realistic time-frame, with a long-term view of the patient's problems.

Management should include ongoing attempts to reach a shared view of expectations, so that neither

\section{Box 4 Features of successful management} plans

Tailored to individual's needs

Explicit goals, clearly formulated

Realistic goals

Prioritised goals

Long-term time-frame

Attempt to reach shared expectations

Consistent approach

Tolerant approach

Multi-disciplinary

the patient nor the therapist is set up to fail. An acceptance that there may be recurrent breakdown in relationships, work and accommodation and that engagement may be difficult should be combined with a continuing commitment to the patient (Dowson \& Grounds, 1995). The problem occurs when the public, and sometimes the patient, place too high an expectation on the psychiatrist to provide instant solutions or to take responsibility for all adverse behaviours. Norton (1996) has described some of the difficulties in achieving a shared realistic set of expectations and ways of tackling them.

Consistency, tolerance, patience and understanding (without necessarily condoning) are key features of any successful management plan. This can be challenging in light of some patients' ambivalence and difficulty engaging. Another key ingredient is the instillation of hope. However, this should not be at the expense of raising false expectations.

A care plan will usually need to be multi-agency and multi-disciplinary. Patients present with

\section{Box 5 Possible management goals}

\section{Support}

Monitoring and supervision

Intervening in crises

Increasing motivation and compliance

Increasing understanding of difficulties

Building a therapeutic relationship

Avoiding deterioration

Limiting harm

Reducing distress

Treating comorbid Axis I disorders

Treating specific areas (e.g. anger, self-harm, social skills, offending behaviour)

Giving practical support with housing finance, child care 
complex difficulties and no single treatment intervention is likely to meet the diverse needs of patients with personality disorders. They may need several types of help, delivered by a team of professionals. Treatment and intervention will not be purely psychological but will also need to include help with the disabling effects on social functioning. Social problems such as housing, finances, employment and family relationships will need to be tackled. Mental health professionals may need to work closely with primary care, social workers and probation officers, many of whom feel poorly supported and isolated in their involvement with patients with personality disorder. Such patients can be very draining to deal with and it helps to spread the burden, leaving no single individual feeling responsible for everything.

\section{Specific interventions}

Although more rigorous research is needed, there is reasonable evidence to suggest that specific interventions may be of benefit in treating certain symptoms and behaviours associated with personality disorders.

Pharmacological treatments show some promise in the treatment of individual symptoms rather than discrete personality disorder categories (for a review see: Sanislow \& McGlashan, 1998; Tyrer, 1998). In particular, lithium may be helpful in the treatment of aggressive and assaultive behaviour. Anticonvulsants are useful in treating mood instability, irritability and impulsivity. Antipsychotics show moderate efficacy in treating the psychotic symptoms sometimes experienced by patients with borderline and schizotypal personality disorder. Selective serotonin reuptake inhibitor (SSRI) antidepressants can be useful in the treatment of patients with borderline personality disorder and those with mood symptoms associated with personality disorders of the anxious, fearful cluster.

A number of different psychological interventions have been reported on (for a review see: Dowson \& Grounds, 1995; Sanislow \& McGlashan, 1998). Those targeted at specific behaviours have produced the best evidence of effectiveness (Sanislow \& McGlashan, 1998). The symptoms and behaviours associated with borderline personality disorders have received the most attention.

One of the most promising psychosocial interventions for patients with borderline personality disorder is dialectical behaviour therapy (DBT) (Linehan et al, 1991; Palmer, 2001, this issue). The treatment targets problems of affect regulation and deficits in interpersonal skills. DBT was found to be better than treatment as usual in reducing parasuicidal behaviours, enabling patients to stay in treatment, reducing time spent in hospital, and improving social and global functioning (Linehan et al, 1991, 1994).

Social skills training and behavioural techniques have been found to be useful in treating the social skills deficits and avoidant behaviours seen in avoidant personality disorder.

Cognitive-behavioural therapy may be of use in targeting the same symptoms and behaviours as is aimed at in wider psychiatric practice, e.g. anxiety and anger. Few studies have looked specifically at its use in personality disorder but it has been suggested that it could be useful in targeting the dysfunctional beliefs that lead to maladaptive behaviours in many individuals with personality disorder (Enright, 1997).

Cognitive-analytic therapy shows some promise in patients with borderline personality disorder but needs further evaluation (Ryle \& Golynkina, 2000; Denman, 2001). Dynamic insight-oriented psychotherapy, with both groups and individuals, has long been used for some patients. Interventions vary in their frequency, length and approach. Finally, socalled 'good clinical care' or supportive psychotherapy (Dowson \& Grounds, 1995) should not be forgotten, even though it has not been rigorously evaluated.

For a small proportion of highly motivated patients, treatment in a therapeutic community can be very effective in reducing both psychopathology and service consumption (Dolan et al, 1996, 1997).

\section{Service models}

The principles outlined above are consistent with the case management approach: namely, a comprehensive assessment of individual need, the development of an individualised package of care to meet the need and the offer of long-term support adjusted according to fluctuating levels of individual need. This sort of assessment and management plan falls within the everyday work that all mental health services are involved in using the Care Programme Approach (CPA). The CPA recommends its use in the care of certain patients with personality disorder. Supervision registers for patients at significant risk of suicide, self-neglect or causing violence were specifically set up to include patients with personality disorders receiving treatment from specialist psychiatric services.

Most research into treatment of personality disorders has looked at specific treatments for specific symptoms. It has not examined the 
effectiveness of different service delivery models, despite the fact that most patients are likely to need multi-disciplinary input (Links, 1998). It has been argued, on the basis of high drop-out rates, that community services do not currently provide a structure that meets the needs of patients with personality disorder (Joseph, 1992).

However, a review of the literature on service delivery models for patients with personality disorders concluded that assertive community treatment (ACT) programmes seem to offer potential (Links, 1998). The effectiveness of ACT with this group parallels that with patients with chronic psychotic disorders. There are declines in hospitalisation, increased reports of satisfaction with the programme and better overall compliance. A major focus of service delivery for such patients has to be tackling the issue of non-compliance. Attention also has to be given to the specific rehabilitation needs of this group of patients and developing effective ways of helping patients to engage in therapy. Work has shown that treatment, in this case DBT, can increase engagement in therapy in populations with severe disorders (Linehan et al, 1991).

Although it is often suggested that hospital admission should be avoided and carries risks to staff and patients, there is evidence that in some circumstances it may still need to be part of an integrated programme of services (Links, 1998). A randomised trial comparing an early-intervention community service with standard hospital treatment for patients presenting to a psychiatric emergency clinic found that patients with personality disorder showed greater improvement in depressive symptoms and social functioning when referred to hospital rather than to the community service (Tyrer et al, 1994).

It is helpful to outline in advance what part admission will play in any management plan. The aim may be to have short admissions under specified circumstances agreed in advance; these might include brief psychotic episodes, crises with potentially damaging long-term consequences and facilitation of diagnosis or treatment planning.

\section{Barriers to effective management}

There are a number of barriers to the effective management of individuals with personality disorder. Difficulties occur because many people with personality disorder have significant difficulties in their interpersonal relationships. This may adversely affect the interaction between patient and mental health professional, making it difficult to maintain an ordinary clinical focus that could identify relevant and achievable goals. Norton (1996) has described some of the principles of overcoming these.

There are a number of reasons why clinicians may avoid making a diagnosis of personality disorder, may inadequately assess individuals with personality disorder and/or reject them from services. Clinicians may fear that they will be held responsible for their patients' behaviour; may be concerned that their resources are too scarce or unsuited to manage a group of patients who are unlikely to engage and have the capacity to disrupt others; may feel overwhelmed by the diagnosis; may lack confidence in their ability to manage such patients; may consider patients untreatable; or may dislike the patient. Patients with personality disorder can engender feelings of frustration, helplessness, anger, rejection and nihilism in those caring for them. Lack of communication between agencies may hamper effective management. Antisocial behaviour may be used as a bar from services. There may be a lack of training. Most important, there may be a failure to take a longitudinal perspective, leading to misdiagnosis. There are instances, however, when, owing to inadequate assessment, difficult behaviour is inappropriately labelled as personality disorder.

Some have gone so far as to argue that the diagnosis of personality disorder should be abandoned because it is an ill-defined concept used to stigmatise people whose behaviour deviates from social norms and to exclude them from services (Lewis \& Appleby, 1988). However, the fact that a diagnosis may be misused is not a reason to abandon it. It is much easier to address the problems mentioned above if a patient has been thoroughly assessed and the problems broken down into manageable pieces.

\section{Conclusions}

Most mental health professionals have considerable experience of, and skills in, managing patients with personality disorder, even though they are rarely explicitly treating the personality disorder itself. The principles of assessment and management are the same as for any other chronic mental disorder. The effective drawing up of a management plan involves a functional analysis: listing problem areas and associated distress and interference with function, prioritising areas for intervention and setting achievable goals, which might be quite modest. Lack of appropriate resources or strong countertransference feelings should not be confused with untreatability. 


\section{References}

Adshead, G. (2001) Murmurs of discontent: treatment and treatability of personality disorder. Advances in Psychiatric Treatment, 7, 407-415.

American Psychiatric Association (1994) Diagnostic and Statistical Manual of Mental Disorders (4th edn) (DSM-IV). Washington, DC: APA.

Casey, P. R. \& Tyrer, P. (1990) Personality disorder and psychiatric illness in general practice. British Journal of Psychiatry, 156, 261-265.

de Girolamo, G. \& Reich, J. H. (1993) Epidemiology of Mental Disorders and Psychosocial Problems: Personality Disorders. Geneva: World Health Organization.

Denman, C. (2001) Cognitive-analytic therapy. Advances in Psychiatric Treatment, 7, 342-256.

Department of Health (2000a) NHS Hospital In-patient Data for the Period of 1 April 1998 to 31 March 1999. London: Department of Health.

Department of Health (2000b) In-patients Formally Detained in Hospitals under the Mental Health Act 1983 and Other Legislation, England: 1989-1990 to 1999-2000. London: Department of Health.

Dolan, B. M., Warren, F. M., \& Menzies, D., et al (1996) Costoffset following specialist treatment of severe personality disorders. Psychiatric Bulletin, 20, 413-417.

- - — \& - (1997) Change in borderline symptoms one year after therapeutic community treatment for severe personality disorder. British Journal of Psychiatry, 171, 274279.

Dowson, J. H. \& Grounds, A. T. (eds) (1995) Personality Disorders, Recognition and Clinical Management. Cambridge: Cambridge University Press.

Emerson, J., Pankrantz, L., Joos, S., et al (1994) Personality disorders in problematical medical patients. Psychosomatics, 35, 469-473.

Enright, S. J. (1997) Cognitive behaviour therapy - clinical applications. BMJ, 314, 1811-1816.

Gunn, J. (1993) Personality disorders. In Forensic Psychiatry: Clinical, Legal and Ethical Issues (eds J. Gunn \& P. J. Taylor) London: Butterworth Heinemann.

Higgitt, A. \& Fonagy, P. (1992) Psychotherapy in borderline and narcissistic personality disorder. British Journal of Psychiatry, 161, 23-43.

Johnson, J. G, Cohen, P., Skodol, A. E., et al (1999) Personality disorders in adolescence and risk of major mental disorders and suicidality during adulthood. Archives of General Psychiatry, 56, 805-811.

Joseph, P. (1992) Non-custodial treatment: can psychopaths be treated in the community? Criminal Behaviour and Mental Health, 2, 192-200.

Kent, S., Fogarty, M. \& Yellowlees, P. (1995) Heavy utilization of inpatient and outpatient services in a public mental health service. Psychiatric Services, 46, 1254-1257.

Lewis, G. \& Appleby, L. (1988) Personality disorder: the patients psychiatrists dislike. British Journal of Psychiatry, 153, 44-49.

Linehan, M., Armstrong, H., Suarez, A., et al (1991) cognitive behavioural treatment of chronically parasuicidal borderline patients. Archives of General Psychiatry, 48, 10601064

Linehan, M. M., Tutek, D. A., Heard, H. L, et al (1994) Interpersonal outcome of cognitive behavioral treatment of chronically suicidal borderline patients. American Journal of Psychiatry, 151, 1771-1776.

Links, P. (1996) Clinical Assessment and Management of Severe Personality Disorder. Washington DC: American Psychiatric Press.

Links, P. S. (1998) Developing effective services for patients with personality disorder. Canadian Journal of Psychiatry, 43, 251-259.

Maden, A., Curle, C., Meux, C., et al (1995) Treatment and Security Needs of Special Hospital Patients. London: Whurr.
Menzies, D., Dolan, B. M. \& Norton, K. (1993) Are short term savings worth long term costs? Funding treatment for personality disorders. Psychiatric Bulletin, 17, 517-519.

Moran, P. (1999) Antisocial Personality Disorder: An Epidemiological Perspective. London: Gaskell.

Nakao, K., Gunderson, J. G., Phillips, K. A., et al (1992) Functional impairment in personality disorders. Journal of Personality Disorders, 6, 24-33.

Norton, K. (1996) Management of difficult personality disorder patients. Advances in Psychiatric Treatment, 2, 202210.

Palmer, R. (2001) Dialectical behaviour therapy for borderline personality disorder. Advances in Psychiatric Treatment, $\mathbf{8}$ $10-16$.

Reich, J. \& Green, A. I. (1991) Effect of personality disorders on outcome of treatment. Journal of Mental and Nervous Diseases, 179, 74-82.

-, Boerstler, H., Yates, W., et al (1989) Utilization of medical resources in persons with DSM-III personality disorders in a community sample. International Journal of Psychiatry in Medicine, 19, 1-9.

Ryle, A. \& Golynkina, K. (2000) Effectiveness of time-limited cognitive analytic therapy of borderline personality disorder: factors associated with outcome. British Journal of Medical Psychology, 73, 197-210.

Sanislow, C. A. \& McGlashan, T. H. (1998) Treatment outcome of personality disorders. Canadian Journal of Psychiatry, 43, 237-250.

Singleton, N., Meltzer, H. \& Gatward, R. (1998) Office of National Statistics Survey of Psychiatric Morbidity Among Prisoners in England and Wales. London: HMSO.

Spence, S. A., \& McPhillips, M. A. (1995) Personality disorder and police Section 136 in Westminster: a retrospective analysis of 65 assessments over six months. Medicine, Science and the Law, 35, 48-52.

Steels, M., Roney, G., Larkin, E., et al (1998) Discharged from hospital: a comparison of the fates of psychopaths and the mentally ill. Criminal Behaviour and Mental Health, $8,37-53$.

Tyrer, P. (1998) Drug treatment of personality disorder. Psychiatric Bulletin, 22, 242-244.

-, Seivewright, N., Ferguson, B., et al (1990) The Nottingham study of neurotic disorder: relationship between personality status and symptoms. Psychological Medicine, 20, 423-431

-, Merson, S., Onyett, S., et al (1994) The effect of personality disorder on clinical outcome, social networks, and adjustment: a controlled trial of psychiatric emergencies. Psychological Medicine, 24, 731-740.

Westen, D. \& Arkowitz-Westen, L. (1998) Limitations of Axis II in diagnosing personality pathology in clinica practice. American Journal of Psychiatry, 155, 1767-1771.

Winston, A. P. (2000) Recent developments in borderline personality disorder. Advances in Psychiatric Treatment, 6, 211-217.

World Health Organization (1992) The ICD-10 Classification of Mental and Behavioural Disorders. Clinical Descriptions and Diagnostic Guidelines. Geneva: WHO.

- (2001) International Classification of Functioning, Disability and Health (ICF). Geneva: WHO.

\section{Multiple choice questions}

1. Personality disorders:

a are not mental disorders

$\mathrm{b}$ are rarely associated with distress

c lead to impairment in functioning

$\mathrm{d}$ have no relevance in the management of other conditions

e can be difficult to manage. 
2. The following statements about the prevalence of personality disorder are true:

a about half of psychiatric in-patients have a personality disorder

b about half of psychiatric in-patients have a primary diagnosis of personality disorder

$c$ about a third of GP attendees have a personality disorder

d no patients assessed under Section 136 have a personality disorder

e $20-40 \%$ of psychiatric out-patients have a personality disorder.

3. Individuals with personality disorder:
a rarely present to services
$\mathrm{b}$ have high rates of Axis I disorders
c have less severe Axis I symptoms
d have an excess mortality
e have high rates of physical morbidity.

4. The following statements about the management of personality disorder are true:

a individuals with personality disorder have a universally poor prognosis

b there is no point treating personality disorder if there is no cure

c all individuals with personality disorder have the same treatment needs

d a functional analysis can be useful in assessing personality disorders

e the CPA approach has no role in the management of personality disorder.
5. The following have been identified as important features of management plans for personality disorders:

a explicit goals

b realistic goals

c prioritised goals

d must always aim for major personality change

e consistency.

\section{Childhood-Onset Eating Problems: Findings from Research \\ By Claudine Fox and Carol Joughin}

Within the past few years, eating problems in childhood have become a real public health issue. Problems at this stage in the life course have a huge potential impact on the growth and development of the child and cause anxiety for parents and carers. This report is an up-to-date information resource and discusses the current position of research within the field with critical appraisal. It contains data about incidence and practical information about interventions and treatments. It also examines aetiological and maintaining factors, assesses long-term effects of an inadequate diet and discusses potential and actual outcomes for the different disorders.

ISBN1 90124276 5, 96pp, paperback, February 2002, Price $£ 12.50$ 